\title{
Free-to-Roll Investigation of Uncommanded Lateral Motions for an Aircraft with Vented Strakes
}

\author{
Elaine M. Bryant \\ United States Air Force /University of Maryland, College Park, Maryland, 20742, USA \\ D. Bruce Owens ${ }^{\dagger}$ \\ NASA Langley Research Center, Hampton, Virginia, 23681, USA \\ and \\ Jewel B. Barlow ${ }^{\dagger}$ \\ University of Maryland, College Park, Maryland, 20742, USA
}

\begin{abstract}
A free-to-roll study of the low-speed lateral characteristics of the pre-production F/A-18E was conducted in the NASA Langley 12-Foot Low-Speed Tunnel. In developmental flight tests the F/A-18E unexpectedly experienced uncommanded lateral motions in the power approach configuration. The objective of this study was to determine the feasibility of using the free-to-roll technique for the detection of uncommanded lateral motions for the preproduction F/A-18E in the power approach configuration. The data revealed that this technique in conjunction with static data revealed insight into the cause of the lateral motions. The free-to-roll technique identified uncommanded lateral motions at the same angle-of-attack range as experienced in flight tests. The cause of the uncommanded lateral motions was unsteady asymmetric wing stall. The paper also shows that free-to-roll data or static force and moment data alone are not enough to accurately capture the potential for an aircraft to experience uncommanded lateral motion.
\end{abstract}

\section{Nomenclature}

$\mathrm{AOA}=$ angle of attack

$C_{L} \quad=$ lift coefficient

$C_{I} \quad=$ total rolling moment coefficient

$C_{l o} \quad=$ rolling moment forcing function coefficient

$C_{l p} \quad=$ dynamic lateral stability coefficient, roll damping

$C_{l B}=$ static lateral stability coefficient, spring effect

FTR $=$ free-to-roll

HT = horizontal tail

LEF = leading edge flap

LEX = leading edge extension

PID $=$ Parameter Identification

psf $=$ pounds per square feet

TEF $\quad=$ trailing edge flap

VT $=$ vertical tail

\footnotetext{
* Graduate Student, Aerospace Engineering, College Park MD, Member AIAA.

+ Aerospace Engineer, Vehicle Dynamics Branch, M/S 153, Associate Fellow, AIAA.

‡ Director Glenn L. Martin Wind Tunnel, Aerospace Engineering, College Park MD, Associate Fellow AIAA.
}

This paper is declared a work of the U.S. Government and is not subject to copyright protection in the United States. "The views expressed in this article are those of the author and do not reflect the official policy or position of the United States Air Force, Department of Defense, or the U.S. Government." SPR-179.04 


$\begin{array}{ll}\alpha & =\text { angle of attack } \\ \beta & =\text { angle of sideslip } \\ \phi & =\text { body axis roll angle }\end{array}$

\section{Introduction}

$\mathrm{I}^{\mathrm{N}}$ 1996 the U.S. Navy's F/A-18E/F Super Hornet experienced uncommanded lateral motions, or wing drop, in the power approach (PA) configuration during developmental flight tests. These motions were not expected based on computational or experimental predictions during the design studies. Wing drop posed a potential risk to flight safety due to its occurrence at low altitudes and airspeeds. Based on data from investigative flight tests, wing drop was eliminated by retracting deployable vents located on the leading edge extension (LEX). The vents were located at the junction of the LEX and the wing. Figure 1 shows the location of the LEX and the LEX vent in the open position. Aligning the LEX vent flush with the the LEX generates a LEX vents-closed configuration. Although the LEX vents are not employed on the production version of the Super Hornet several efforts have been made to understand how opening the vents changed the flow topology

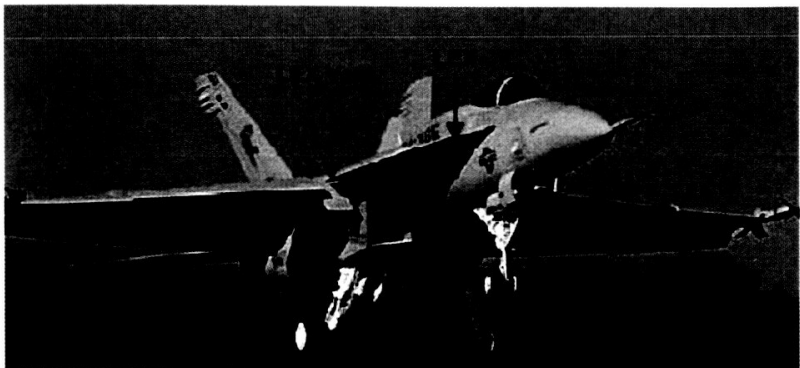

Figure 1. F/A-18E Super Hornet in approach configuration with the LEX vents open ${ }^{5}$ that resulted in the uncommanded motions

Cook and Barlow ${ }^{2-4}$ and Cook ${ }^{1}$ reported studies of the flow topologies on pre-production F/A-18E models that have shed much light on the characteristics of the flows. Some aspects of time dependence of forces and flows have been observed and reported but the models have always been held stationary. In actual flight events, the airplane exhibited transient motions in response to force and moment variations due to wing flow unsteadiness. This experience provides a strong motivation for the application of the free-to-roll (FTR) method. The classical FTR test technique offered promise for shedding additional light on the flight dynamics and aerodynamics of the lateral motions of the vents-open and vents-closed pre-production F/A-18E configurations. Owens et $\mathrm{al}^{5,6}$ reported on an extensive study of transonic uncommanded lateral motions of military aircraft using the free-to-roll technique, including the pre-production F/A-18E. The motivations, methods, and objectives for the application of the FTR method to the PA configuration parallel those for the transonic FTR tests of the pre-production F/A-18E. The physics of the aerodynamic phenomena associated with the uncommanded motions in the two cases are different.

The objective of this effort was to conduct a FTR feasibility test on the pre-production F/A-18E/F to determine if this technique can be used to detect the uncommanded lateral motions as seen in flight. The method-of-evaluation was to compare indications for uncommanded lateral motions from the FTR method to the results from the in-flight tests (Table 1) and the static data ${ }^{1-4}$. Good correlation between the developmental flighttesting and the FTR testing, supported by static testing, would help establish capability for early identification of potential uncommanded lateral

\begin{tabular}{|c|c|c|c|c|}
\hline $\begin{array}{l}\text { Aircraft, Flight, } \\
\text { and Maneuver }\end{array}$ & $\begin{array}{c}\text { Angle of } \\
\text { Attack (deg) }\end{array}$ & $\begin{array}{c}\text { Angle of } \\
\text { Sideslip (deg) }\end{array}$ & $\begin{array}{l}\text { Leading Edge Flap } \\
\text { Position (deg) }\end{array}$ & $\begin{array}{l}\text { Trailing Edge Flap } \\
\text { Position (deg) }\end{array}$ \\
\hline \begin{tabular}{|l}
$E 1,12,19$ \\
\end{tabular} & 15.0 & 0.0 & 30 & 40 \\
\hline \begin{tabular}{|l}
$E 2,17,67$ \\
\end{tabular} & 12.1 & -0.4 & 26 & 40 \\
\hline$E 1,26,18$ & 158 & 0.2 & 30 & 30 \\
\hline$E 1,26,20$ & 150 & 0.7 & 30 & 40 \\
\hline$E 2,39,13$ & 14.5 & 0.5 & 28 & 40 \\
\hline \begin{tabular}{|l}
$E 2,39,21$ \\
\end{tabular} & 136 & -2.5 & 15 & 30 \\
\hline E2,39,23 & 138 & 1.2 & 15 & 40 \\
\hline$E 2,39,24$ & 13.7 & 0.0 & 15 & 40 \\
\hline E2, 40, 41 & 133 & -1.2 & 27 & 40 \\
\hline $\mathrm{Fl}, 06,12$ & 142 & Not avaulable & 28 & 40 \\
\hline
\end{tabular}

Table 1. Flight test results showing $\alpha$-range of uncommanded lateral motion ${ }^{3}$. motions. In support of this method-of-evaluation a candidate FTR figure-of-merit (FOM) was proposed and assessments were carried out in three areas: severity and types of model motions, unsteady and nonlinear aerodynamics, and roll damping. 


\section{Experimental Approach}

The model used for both the static and the FTR testing was a $10 \%$ scale model of the pre-production F/A-18E. The model, constructed of balsa wood, plywood, fiberglass and aluminum, was outfitted with wing tip missiles, canopy, engine inlets, leading edge flaps (LEF), ailerons, flap shrouds, trailing edge flaps (TEF), vertical tail (VT), horizontal tail (HT) and LEX vents. The control surfaces were movable and could be set at specific values. For the data presented herein the HTs and rudders were set to $0^{\circ}$. The LEX vents could be set to various open positions or they could be closed completely. The static and FTR testing were both done in the NASA Langley 12-Foot LowSpeed Tunnel. The experiments were conducted at sea-level pressure and density with a freestream dynamic pressure of $4 \mathrm{psf}$ resulting in a mean aerodynamic chord-based Reynolds number of $0.5 \times 10^{6}$. Although numerous configurations were tested, the paper will present LEX vents closed and LEX vents open with the LEF $=10^{\circ}, T E F=$ $30^{\circ}$, and ailerons $=30^{\circ}$ (symmetric trailing-edge-down deflection). This positioning of the ailerons and TEF is referred to as the PA-half configuration. It is a slight modification from the PA configuration used in flight tests.

\section{A. Static Testing}

The static force and moments were measured using an intemally mounted six-component strain gauge balance (NASA FF12). A series of both $\alpha$ - and $\beta$-sweeps were conducted with the configurations. Initially $\alpha$-sweeps over the range $-4^{\circ} \leq \alpha \leq 20^{\circ}$ were conducted but for the majority of the runs a smaller $\alpha$-range $\left(10^{\circ} \leq \alpha \leq 20^{\circ}\right)$ with a higher resolution was chosen to cover the area of interest. Also, $\beta$-sweeps with a range of $-16^{\circ} \leq \beta \leq 16^{\circ}$ were performed in the smaller $\alpha$-range to assess the static lateral characteristics. The data were sampled at a rate of $80 \mathrm{~Hz}$ for 10 seconds using a low-pass analog filter with cutoff frequency of $4 \mathrm{~Hz}$. All data taken during the 10 second sample time was recorded. The paper will present both the time-averaged balance data as well as the time history of the balance signals over this 10 second window.

\section{B. Free to Roll Testing}

In the FTR test technique the model is constrained to roll about the longitudinal body axis. Switching from the static-force-and-moment phase to the FTR requires replacing the balance with the FTR rig. Modifications to the interior of the model were required in order to accommodate the FTR rig. The FTR rig houses a resolver to measure the roll angle time history with an accuracy of 0.12 degrees. The roll angle signal was recorded at a rate of $200 \mathrm{~Hz}$ using a low-pass analog filter with a cutoff frequency of $4 \mathrm{~Hz}$. Video of the lateral activity was also recorded. The FTR rig contains an air brake to stop the motion and allow the data point to start with a zero initial roll rate. The model's mass was balanced such that the lateral and vertical center-of-gravity coordinates were located on the roll axis. The model's roll inertia was determined experimentally and found to be $0.40 \mathrm{slug}-\mathrm{ft}^{2}$. This inertia value is close to that required for dynamic scaling. With knowledge of the roll inertia, the total rolling moment, $C_{l}$, can be calculated by twice differentiating the roll angle time histories. The rolling-moment time histories are then used in PID methods to determine $C_{l p}$. The PID method used is described in Ref. 5.

There were three ways to conduct a FTR test point: continuous pitch sweeps, pitch-pause, and bank \& release. For the continuous pitch-sweeps, the model was allowed to roll freely while going through a range of pitch angles. This type of test point quickly reveals any lateral activity over the $\alpha$-range. The procedure for a pitch-pause point involved setting the model to the desired $\alpha$ and holding it there with the brake. Upon brake release the lateral motion was recorded. The information gathered reveals what the model will do when the roll angle and the roll rate are set to zero. The procedure for the bank \& release points was to set the model at an initial roll angle other than zero and then release the brake. The bank $\&$ release points are used to assess how the model will react to a given initial rolling moment, assess roll-damping for the cases where no lateral activity existed at a pitch-pause point, and investigate how inducing a rolling motion affects any motions observed previously.

In order to quantify the lateral activity a FOM was used similar to the one developed by Owens et $a^{5,6}$. The FOM is calculated from the roll-angle time histories and captures amplitude and rate effects. It is defined by:

$p_{P-l} \equiv\left(\left|\frac{\Delta \phi}{\Delta t}\right| \frac{b}{2 V_{\infty}}\right)_{\max }$. The plots of the FTR-FOM versus $\alpha$ were used to quantify the relative severity of the lateral activity for various configurations and test conditions. This FOM is not intended to indicate the type of motion, how long it took for the motion to develop or how often the events happened. However it has proven to be an accurate indicator of where uncommanded lateral motion will occur in flight ${ }^{5}$ and serves as conservative first filter for assessing the severity of the motion.

The FTR roll angle time histories must be interpreted as open-loop motions due to aerodynamics and cannot be directly used to predict aircraft motions. The rolling motion can be described by a combination of forcing functions, 
roll damping $\left(C_{l p}\right)$, and static lateral stability $\left(C_{\mid \beta}\right)$ effects. Therefore, it is instructive when analyzing the data from a FTR test to consider the equation of motion in terms of the Euler angle $\phi$ as: $\frac{I_{x} \ddot{\phi}}{q S b}+C_{l_{\dot{\phi}}} \frac{\dot{\phi} b}{2 V_{\infty}}+C_{l_{\phi}} \phi=C_{l_{o}}$. The foregoing equation is in the form of the classical mass-spring-damper system where: $C_{l o}$ represents an aerodynamic forcing function; $C_{l_{\phi}}$ represents the spring constant which, along with the inertia, determines the frequency of oscillation; and $C_{l_{b}}$, represents the damping coefficient. In the FTR technique, the use of $C_{l_{\dot{\phi}}}$ and $C_{l p}$ is kinematically equivalent. By measuring roll angle versus time, the FTR technique captures the composite effect of both static and dynamic forces acting on the model regardless of whether they are steady or unsteady.

\section{Results and Analysis}

This section will discuss the results for the vents-open and vents-closed configurations. The analysis will begin by using the FTR-FOM to show the relative severity and $\alpha$-ranges of lateral activity for the configurations. Then detailed analysis will be presented for representative points within the $\alpha$-range of lateral activity. The lateral activity of the two configurations is compared in Fig. 2 using the FTR-FOM. The plot shows that for $12^{\circ} \leq \alpha \leq 15^{\circ}$ opening

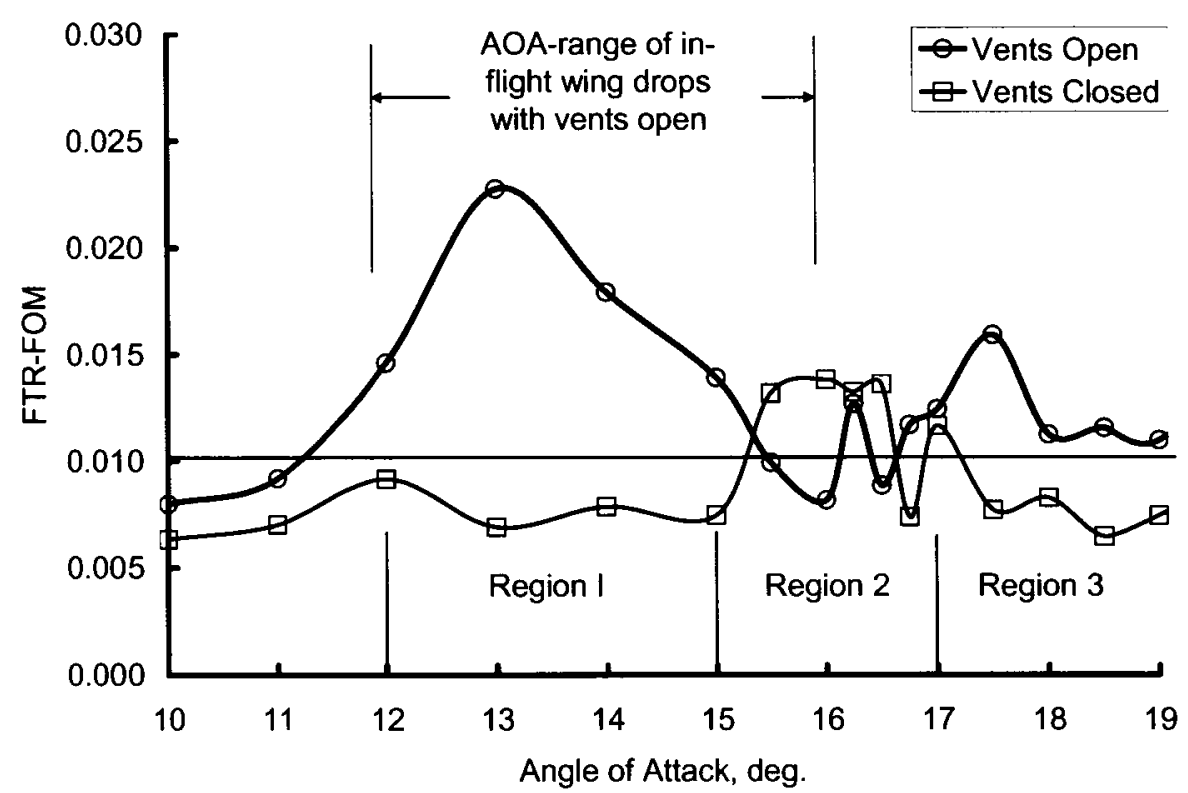

Figure 2. FTR-FOM comparison between vents closed and vents open. Values of the FOM are calculated using pitch-pause points. the vents causes a significant increase in lateral activity with the maximum activity being at $\alpha=13^{\circ}$. This $\alpha$-range directly correlates to the $\alpha$-range in flight tests where wing drop occurred ${ }^{5}$ (Table 1). Significant lateral activity was defined for this test to occur when the motion would produce a value of $p_{p-v} \geq 0.010$. The correlation to flight data using the FTR-FOM is only the $\alpha$-range. For $\alpha=15.5^{\circ}$, $16^{\circ}$, and $16.5^{\circ}$, closing the vents increases lateral activity relative to the vents open position. For $\alpha=16.25^{\circ}$ and $17^{\circ}$, the severity of lateral activity is the same for both positions of the vents. For $\alpha=16.75^{\circ}$

and $\alpha \geq 17.5^{\circ}$ opening the vents caused an increase in lateral activity. In summary, the FTR-FOM plot divides the $\alpha$-range into three regions: $12^{\circ} \leq \alpha \leq 15^{\circ}, 15.5^{\circ} \leq \alpha \leq 17^{\circ}$, and $\alpha>17^{\circ}$. For regions 1 and 3 there is a distinct reduction in lateral activity by closing the vents. In region 2 there are mixed results.

The following discussion will use the FTR-FOM plot as the starting point for more detailed analysis. The points that will be analyzed will be one from region 1 and one from region 2 since this covers the $\alpha$-range where wing drop was seen in flight. From region $1, \alpha=13^{\circ}$ is chosen for analysis since this point shows the largest difference between vents open and closed, and wing drop was first identified in flight at $\alpha=13^{\circ}$. In order to show the amplitude and frequency change of the lateral activity between the two configurations, the roll angle time histories are shown for vents open and closed in Fig 3. The plot reiterates the large reduction in lateral activity by closing the vents. The cause of the rolling motion can be generated by any or all combinations of a forcing function, $C_{l o}$, spring effects, $C_{l B}$, and roll damping, $C_{l p}$. Also, the aerodynamic terms may be unsteady. The following discussion will address each of these possibilities. 


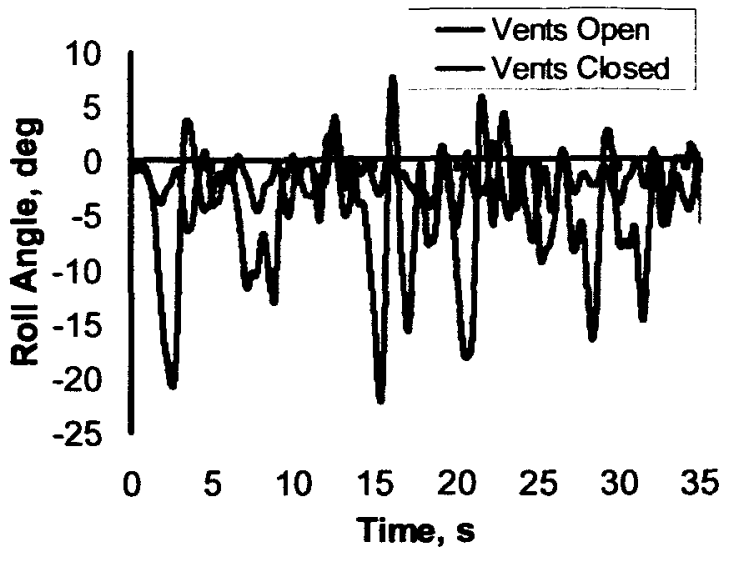

Figure 3. Roll angle time histories for the ventsopen and closed configurations at $\alpha=13^{\circ}$.

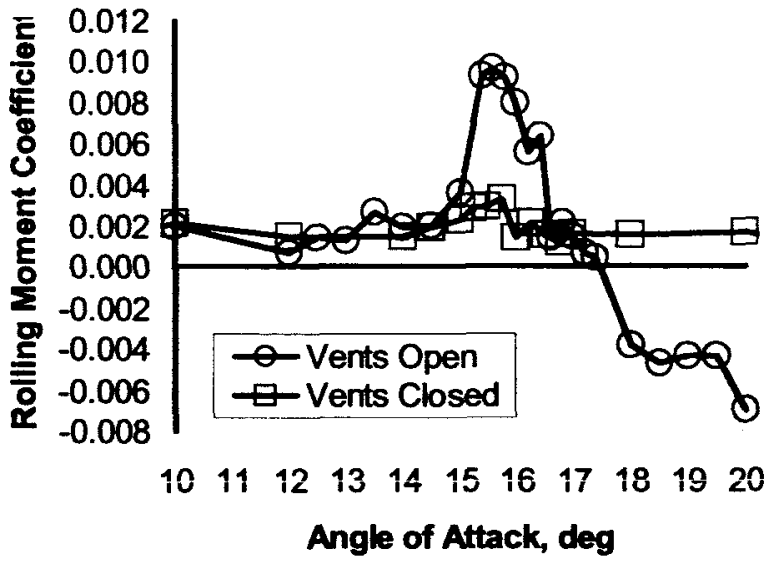

Figure 4. Effect of vents on the time-averaged static rolling moment.

The time-averaged values of $C_{l o}$ are shown in Fig. 4 with a plot of rolling moment coefficient from the static force \& moment test. (Note. There is an average $C_{1}=0.002$ offset for both configurations below $\alpha=17^{\circ}$. The cause of this asymmetry in the data is probably due to tunnel sidewash and/or model asymmetries. The data will be

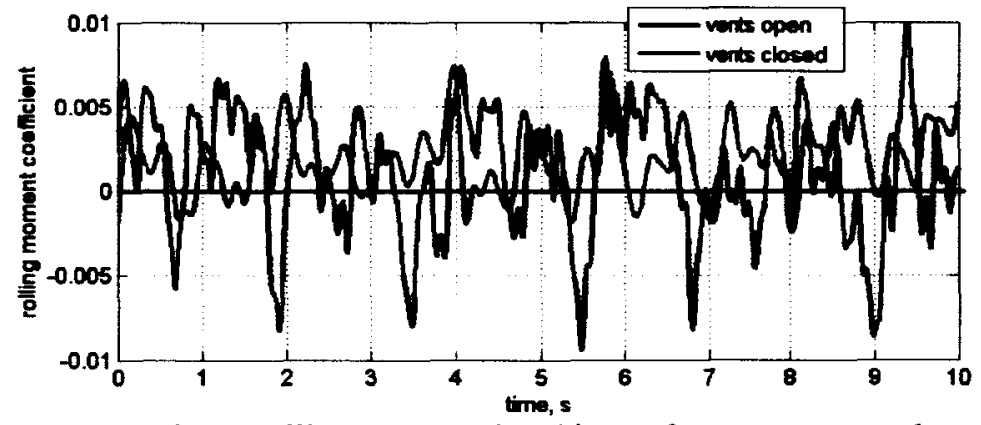

Figure 5. Balance rolling moment time history for vents open and vents closed, $\alpha=12^{\circ}$. discussed relative to the offset.) The time-averaged value of $C_{10}$ at $\alpha=13^{\circ}$ for the vents-open configuration is not large enough to cause the significant lateral activity that was seen on the FTR rig. Balance rolling moment time histories indicate that $C_{l o}$ is unsteady for the vents-open position. The time history signals for the $\alpha=12^{\circ}$ data in Fig. 4 is shown in Fig. 5 for the ventsopen and -closed position. The timeaverage of these signals is shown in Fig. 4 at $\alpha=12^{\circ}$. No balance data was taken for the vents-closed case at $\alpha=13^{\circ}$ so a comparison is made at $\alpha=12^{\circ}$. The characteristics of the balance signals at $\alpha=12^{\circ}$ is indicative of the balance signals where data was taken in the $12^{\circ} \leq \alpha \leq 15^{\circ}$ for the two configurations. The plot shows that the vents-open configuration has aperiodic rolling moment spikes with sufficient amplitude and low enough frequency that would probably cause rigid body rolling motion. Figure 6 shows the static lateral stability characteristics, $C_{l \beta}$, for the ventsopen configuration. The plot shows that the model has a strong spring, $C_{l \beta}$, which contributes to the lateral activity seen in the roll angle time history plot of Fig. 3. The lift characteristics of the two configurations are shown in Fig. 7. The data show that the vents-open configuration produces a more non-linear lift curve than the vents-closed configuration. Nonetheless, there are no sharp breaks with significant loss in lift. These data show that even though no time-averaged rolling moment spike occurs (Fig. 4) and no significant changes in the time-averaged lift curve slope are evident (Fig. 7), the wing can still experience an unsteady aerodynamic forcing function that causes uncommanded lateral motions. It is then left to the FTR technique to show that this unsteady aerodynamic forcing function produces undesirable lateral activity. The roll damping characteristics are shown in Fig. 8 . The plot shows no significant difference or change in roll damping characteristics over the $\alpha$-range where activity was seen in flight. Therefore, roll damping is not a cause of the lateral activity. Therefore, based on the spring-mass-damper analogy, the lateral activity produced by opening the vents in the $12 \leq \alpha \leq 15^{\circ}$ range is caused by a strong spring and an unsteady forcing function. 


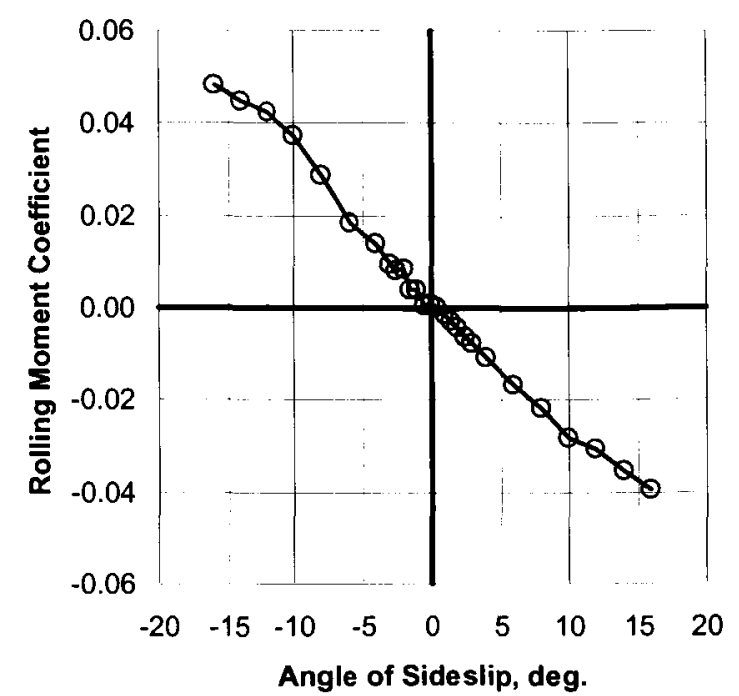

Figure 6. Static lateral stability characteristics for vents-open configuration, $\alpha=13^{\circ}$.

From region 2 , the lateral activity at $\alpha=16^{\circ}$ will be analyzed since this occurs at a critical state (i.e. rapid change in the slope of an aerodynamic parameter) as indicated in the static rolling moment curve of Fig. 4 and is the point in region 2 where the lateral activity between the two configurations is a maximum as indicated by the FTRFOM plot of Fig. 2. The roll angle time histories of the vents-closed and -open positions are shown in Fig. 9. Prior to the FTR testing it was expected that the vents-open position would exhibit significant wing drop at $\alpha$ 's around $16^{\circ}$ because the wing is going through stall and there is a significant spike in the rolling moment curve (Fig. 4). The wing stall (Fig. 7) and rolling moment characteristics (Fig. 4) are benign for the vents-closed configuration. Therefore, it was expected that the vents-closed configuration would not exhibit significant lateral activity. Figure 9 shows with a plot of the roll-angle time histories that just the opposite happened. Compared to vents-open activity at $\alpha=13^{\circ}$, the vents-closed activity at $\alpha=16^{\circ}$ is of smaller amplitude and higher frequency. This is reflected in the FTR-FOM plot, Fig.2.
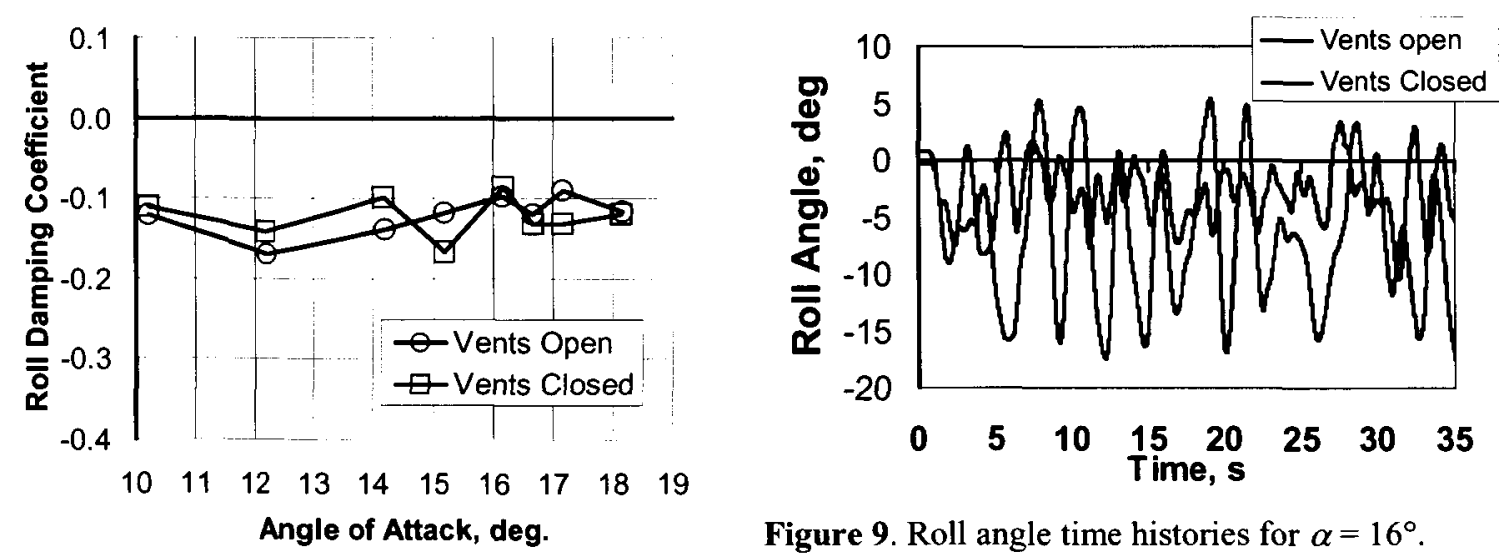

Figure 9. Roll angle time histories for $\alpha=16^{\circ}$.

Figure 8. Effect of vents on roll damping.

The time-averaged value of $C_{l o}$ shown in Fig. 4 shows that at $\alpha=16^{\circ}$ there is a significant value of $C_{l o}(0.006)$ for the vents-open case. The time-history plot for the vents-open configuration (Fig. 9) shows a left wing down bias which contradicts the rolling moment data of Fig 4 . Previous data ${ }^{3}$ shows that this same $10 \% \mathrm{~F} / \mathrm{A}-18 \mathrm{E}$ model can exhibit negative rolling moment spikes. Evidently, when the model was changed from the static force \& moment mount to the FTR the model had a tendency to stall such that a left wing down rolling moment was generated as in the previous test of Ref 3 . 


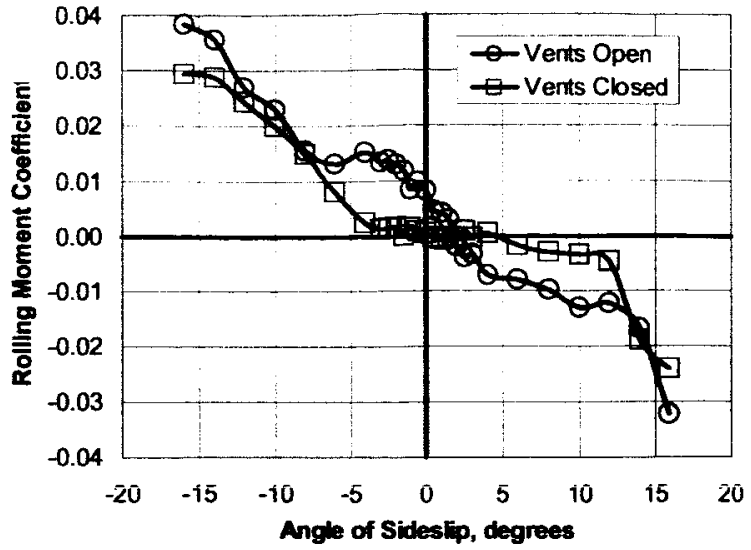

Figure 10. Static lateral stability for vents open and closed at $\alpha=16^{\circ}$.
The static lateral stability at $\alpha=16^{\circ}$ is shown in Fig. 10 with a plot of $C_{l}$ vs $\beta$. The plot shows that the ventsopen configuration is stable in the $-4^{\circ} \leq \beta \leq+4^{\circ}$ range with a $C_{l o}$ of 0.008 . This value equals the $C_{l o}$ in Fig. 4. The trim point is around $\beta=1.75^{\circ}$. This is close to the average value of $\beta$ that corresponds to the average roll angle in Fig. 9 assuming the possibility that the ventsopen curve of Fig. 9 can be reflected about the origin. Figure 10 shows that the vents-closed configuration is neutrally stable with a zero rolling moment value for $-4^{\circ} \leq \beta \leq+4^{\circ}$. Therefore any disturbance in the flow will cause the model to roll within this $\beta$-range. Although not shown, the $\beta$-range for the vents-closed roll angle time history of Fig. 9 is also $-4^{\circ} \leq \beta \leq+4^{\circ}$. Based on the lift characteristics of Fig. 7 and roll damping of Fig. 8 these aerodynamic terms, $C_{L}$ and $C_{l p}$, are not significant causes for the lateral motion of either the vents-open or ventsclosed position. Figure 11 compares the balance rolling-

moment time history of the vents open to vents closed at $\alpha=16^{\circ}$. Figure 11 shows that both vents-open and ventsclosed configurations exhibit unsteady rolling moment variations - though at reduced levels than seen in the previous data for $\alpha=12^{\circ}$. For the case of the vents-closed configuration, the neutral static lateral stability at small $\beta$ s allows the unsteady rolling moment to significantly roll the model. The stable $C_{i \beta}$ characteristic of the ventsopen configuration provides a strong restoring force which decreases the impact of the relatively small unsteady rolling moments (Fig. 11) on motions of the model (Fig. 9), while still exciting small motions about the trim roll angle. The data show that neither the static lateral stability, or the presence of unsteady aerodynamic rolling moments by themselves predict the motion of the model. The FTR testing shows the impact of the combinative effects of static stability, damping, and unsteady aerodynamic forces.

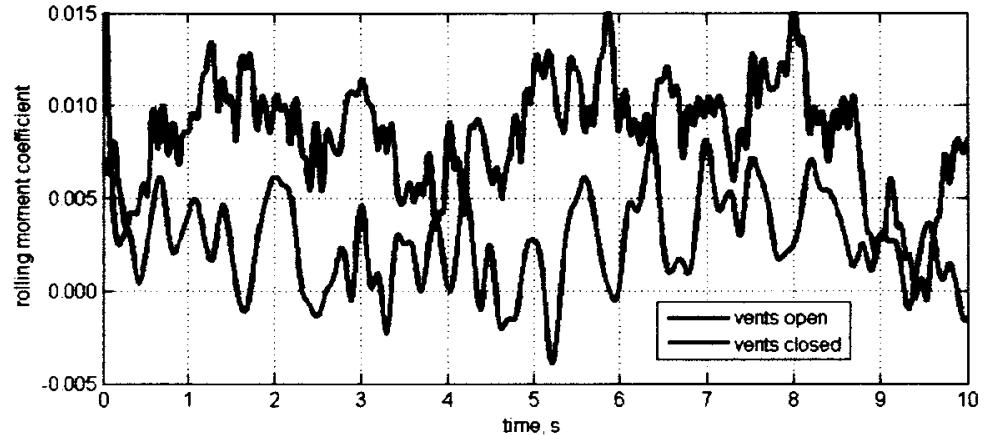

Figure 11. Balance rolling moment time history for vents open and vents closed, $\alpha=16^{\circ}$.

\section{Conclusion}

The FTR technique was used to assess the potential for a pre-production F/A-18E model to predict wing drop as was experienced in flight. Using a FTR-FOM, the technique accurately captured the $\alpha$-range where wing drop was experienced in flight. Also, the technique showed that, in agreement with flight data, lateral activity was significantly reduced if not eliminated by closing the LEX vents. In the $\alpha=12^{\circ}$ to $15^{\circ}$ range, the analysis of the FTR and static force \& moment data showed that the lateral activity with the vents open was caused by an unsteady aerodynamic forcing function and a strong spring. The lift characteristics and time-averaged static rolling moment values showed no indication for wing drop in this $\alpha$-range. This result shows the importance in using the FTR technique along with unsteady force and moment measurements to predict areas of uncommanded lateral motions. In the $15^{\circ}<\alpha<17^{\circ}$ range, the lateral activity of the vents-closed case was primarily caused by neutral static stability with contribution from an unsteady forcing function. In this $\alpha$-range for the vents-open case, the lift characteristics and time-averaged static rolling moment values would indicate a potential for wing drop. The FTR technique showed that the model would just roll over to trim out the rolling moment spike and oscillate with low amplitude and rate about this trim point probably because of an unsteady forcing function. Roll damping was found to be stable for all configurations and therefore was not a contributor to the uncommanded lateral motions. The FTR 
technique can be used to predict areas of uncommanded lateral motions for the pre-production F/A-18E in the PA configuration.

\section{References}

'Cook, S., "On Flow Topology Changes Accompanying Aerodynamic Bifurcation for Aircraft with Vented Strakes", Ph.D. Dissertation, University of Maryland, 2003.

${ }^{2}$ Cook, S., and Barlow, J., "An Investigation of Low-Speed Lateral Characteristics of a Simplified F/A-18E Configuration", AIAA-2003-0931, January 2003.

${ }^{3}$ Cook, S. and Barlow, J., "Investigation of Effects of Leading Edge Extension Vents the Lateral Characteristics of the F/A-18-E in Power Approach Configuration", AIAA 2000-4510, August, 2000.

${ }^{4}$ Cook, S., and Barlow, J., "Investigation of Critical States of the F/A-18E in Power Approach Configuration Using Mini-Tuft Flow Visualization", AIAA 2001-4145, August, 2001.

${ }^{5}$ Owens, B., Capone, F., Hall, R., Brandon, J., and Chambers, J., "Transonic Free-To-Roll Analysis of Abrupt Wing Stall on Military Aircraft," Journal of Aircraft, Vol. 41, No. 3, 2004, pp. 474-484.

${ }^{6}$ Owens, B., McConnell, J., Brandon, J., and Hall, B., "Transonic Free-To-Roll Analysis of the F/A-18E and F-35 Configurations," AIAA 2004-5053, August 2004. 